\title{
Effect of the Magnetic Field on the Flow of a Liquid Metal and Heat Transfer in a Curved Cylindrical Annular Duct with Uniform Wall Temperatures
}

Panteleimon A. Bakalis ${ }^{*}$ and Pavlos M. Hatzikonstantinou

Department of Mechanical Engineering and Aeronautics, University of Patras, 26504, Greece

\section{Abstract}

The laminar fully developed magnetohydrodynamic (MHD) and thermal flow of a liquid metal into a curved annular duct of circular cross section, subjected to a transverse external magnetic field, is studied in the present work. The cylinders are maintained at different uniform temperatures, thus buoyancy forces between the walls are created. Applying our computational methodology, computational results are obtained for different values of curvature (0-0.2), Reynolds number (100-2000), Grashof number (0 $\left.10^{6}\right)$ and Hartmann number (0-2000). The magnitude of the velocity is determined by the balance of the centrifugal, buoyancy and electromagnetic forces.
Publication History:

Received: July 04, 2016

Accepted: December 26, 2016

Published: December 28, 2016

\section{Keywords:}

Magnetohydrodynamics (MHD), Curved Duct, Numerical Analysis, CVP Method

\section{Introduction}

MHD flows involve the motion of an electrically conducting fluid under the effect of an external strong magnetic field and are met in many practical applications such as fusion reactors, electromagnetic pumping and other engineering applications. Thus, different models have been developed for the solution of the Maxwell equations involving different formulations for the induced magnetic field, e.g. [1-7].

The present project involves a geometry with a curved annular duct, as similar geometries are involved in the access ducts of intercooling systems of the dual-coolant breading blankets where lead-lithium (PbLi) is used as the breeder material [8]. Other works involving laminar MHD flows in curved ducts have been performed by Tabeling and Chabrerie [9] at a square channel using an approximate analytical perturbation method and by Issacci et. al [10] in a circular channel with an approximate analytical method. Moresco and Alboussière [11] studied experimentally the instability conditions for the Hartmann layer for the MHD flow on a closed toroidal loop of a square curved duct. The same problem has been studied numerically by Vantieghem and Knaepen [10]. Krasnov et al. [12] studied the problem of linear stability to exponentially growing pertubations in the MHD flow within straight orthogonal ducts. Hoque and Alam [13] studied numerically the MHD flow through a curved pipe of a circular crosssection, under the effect of an axial magnetic field, using a spectral method.

One of the most important issues in the study of the MHD flow is the accurate determination of the induced magnetic field in order the divergence free condition to be satisfied with the highest accuracy. On the previous studies, the divergence of the induced magnetic field was checked to be very small without corrections [14], or it was improved occasionally using numerical or mathematical technicalities [15], without to produce a method for the systematic improvement of the accuracy of the calculation of the induced magnetic field. These problems are related with the fact that the divergence free condition is a first order partial differential equation which cannot incorporates the set of the required boundary conditions in closed space regions. Thus, for the first time in literature, a variational methodology has been developed, which always satisfies the divergence free condition with high accuracy $[16,17]$. The aforementioned methodology is used in conjunction with the b-formulation which is applied for moderate values of the external magnetic field, taking into account all the components of the induced magnetic field. However, as the external magnetic field is increased to higher values, the h-formulation [18] is preferred, where only the axial component of the induced magnetic field is taking into account, whereas the magnitude of its secondary components is reduced dramatically. In the case of extremely high values of the external magnetic field, the reduced formulation ( $r-f$ ) is applied where only the axial components of the velocity and the induced magnetic field are taking into account, because either the secondary velocity components become negligible.

\section{Problem Formulation}

We consider a curved annular duct with radius of curvature $R_{\mathrm{c}}$ and with hydraulic radius $R=R_{\mathrm{o}}-R_{\mathrm{i}}$, where $R_{\mathrm{o}}$ and $R_{\mathrm{i}}$ are, respectively, the radius of the outer and the inner cylinder, with respect to a Cartesian reference system $\left(x^{\prime}, y^{\prime}, z\right)$ of Fig. 1 . The liquid metal is subjected to the action of an external magnetic field $\mathrm{B}_{\mathrm{o}}$ shown in Figure 1 and the inner and outer cylinders are maintained at uniform temperatures, with the inner wall temperature $T_{i}$ to be lower than the outlet wall temperature $T_{\mathrm{o}}$.

The MHD and thermal flow is governed by the following set of nondimensional equations:

$$
\begin{aligned}
& \text { Momentum equation: } \\
& \vec{\partial} v / \partial t+(\vec{V} \cdot \vec{\nabla}) \vec{V}=-\vec{\nabla} P+G r / \operatorname{Re}^{2} \vec{T}_{b}+1 / \operatorname{Re} \cdot \nabla \vec{V}+H a^{2} / \operatorname{Re}^{j \times \vec{B}}(1) \\
& \text { Continuity equation: } \\
& \vec{\nabla} \cdot \vec{V}=\mathrm{O} \\
& \text { Energy equation: } \\
& \partial T / \partial t+(\vec{V} \cdot \vec{\nabla}) T=1 / \operatorname{RePr} \cdot \Delta T \\
& \text { Transport of magnetic induction: }
\end{aligned}
$$$$
\partial \vec{B} / \partial t+(\vec{V} \cdot \vec{\nabla}) \vec{B}=1 / R_{m} \cdot \Delta \vec{B}+(\vec{B} \cdot \vec{V}) \vec{V}
$$

Corresponding Author: Dr. Panteleimon A. Bakalis, Department of Mechanical Engineering and Aeronautics, University of Patras, 26504, Greece; E-mail: pbakalis@teemail.gr

Citation: Bakalis PA, Hatzikonstantinou PM (2016) Effect of the Magnetic Field on the Flow of a Liquid Metal and Heat Transfer in a Curved Cylindrical Annular Duct with Uniform Wall Temperatures. Int J Mech Syst Eng 2: 120. https://doi. org/10.15344/2455-7412/2016/120

Copyright: (c) 2016 Bakalis et al. This is an open-access article distributed under the terms of the Creative Commons Attribution License, which permits unrestricted use, distribution, and reproduction in any medium, provided the original author and source are credited. 
Citation: Bakalis PA, Hatzikonstantinou PM (2016) Effect of the Magnetic Field on the Flow of a Liquid Metal and Heat Transfer in a Curved Cylindrical Annular Duct with Uniform Wall Temperatures. Int J Mech Syst Eng 2: 120. https://doi.org/10.15344/2455-7412/2016/120

Page 2 of 11

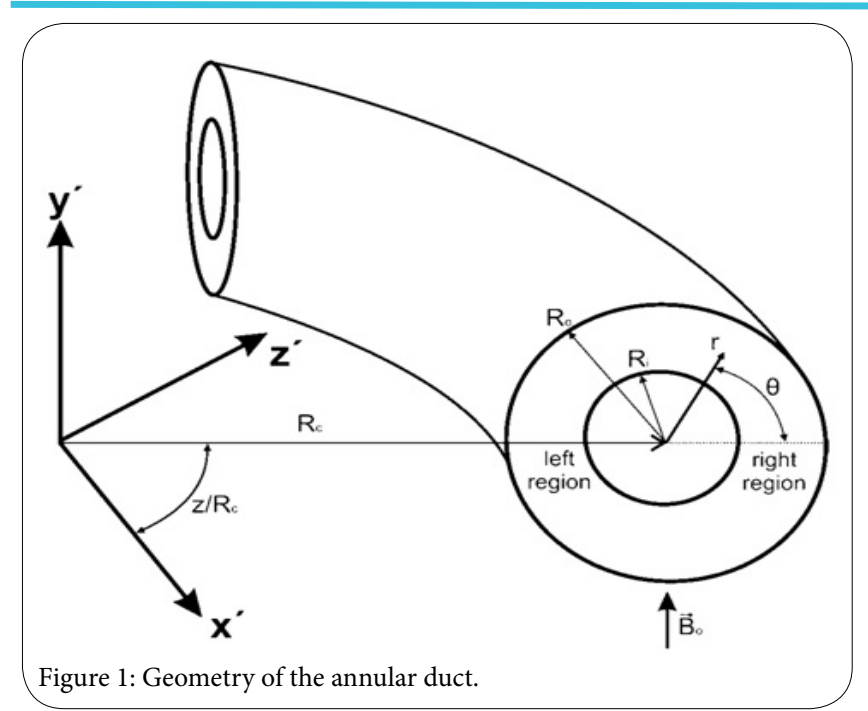

Divergence free equation for the magnetic field:

$\vec{\nabla} \cdot \vec{B}=0$

Ohm's law:

$\vec{J}=-\vec{\nabla} \Phi+\vec{V}+\vec{B}$

Amperes law:

$\vec{J}=1 / R_{m} \vec{\nabla} \times \vec{B}$

Divergence free equation for the electric current density:

$\vec{\nabla} \cdot \vec{J}=0$

where $\vec{V}(r, \theta, z)$ is the velocity, $P=P_{m}+P_{h}$ is the total pressure, $P_{m}$ and $P_{h}$ are, respectively, the magnetic and hydrodynamic pressure, $\vec{T}_{b}=(T \sin \theta, T \cos \theta, 0)$ is the temperature vector field of the buoyancy force term, $\vec{B}=\vec{B}_{o}+\vec{B}_{i}$ is the total magnetic field, $\vec{B}_{o}$ and $\vec{B}_{i}$ are, respectively, the external and the induced magnetic field, $\vec{J}$ is the electric current density, and $\Phi$ is the potential of the electric field. In the energy equation, the effects of viscous dissipation and Joule heating are neglected.

The above equations became dimensionless using the following scales

$$
\begin{gathered}
t=\frac{t^{\prime} V_{0}}{R_{o}^{\prime}}, \vec{r}=\frac{\vec{r}^{\prime}}{R_{o}^{\prime}}, z=\frac{z^{\prime}}{R_{o}^{\prime}}, R_{c}=\frac{R_{c}^{\prime}}{R_{o}^{\prime}}, \vec{V}=\frac{\vec{V}^{\prime}}{V_{0}}, \\
P=\frac{P^{\prime}}{\rho V_{0}^{2}}, T=\frac{T^{\prime}-T_{i}^{\prime}}{T_{o}^{\prime}-T_{i}^{\prime}} \vec{B}=\frac{\vec{B}^{\prime}}{B_{0}^{\prime}}, \vec{J}=\frac{\vec{J}^{\prime}}{\sigma V_{0} B_{0}^{\prime}}
\end{gathered}
$$

where the prime "' " denotes the dimensional variables, and also using the following parameters: the Reynolds number $R e=V_{0} R_{o}^{\prime} / v$, the Hartmann number $H a=\sqrt{\sigma / \rho v} B_{0}^{\prime} R_{o}^{\prime}$, the Grashof number $G r=$ $g \beta \Delta \mathrm{T} R_{o}^{\prime 3} / v^{2}$, the Prandtl number $P r=V / a$ and the magnetic Reynolds number $R_{m}=\mu \sigma V_{0} R_{0}^{\prime}$ where $\rho$ is the fluid density, $v$ is the kinematic viscosity of the fluid, $g$ is the gravitational acceleration, $\Delta T$ is the temperature difference between the walls, $a$ is the thermal diffusivity, $R_{o}^{\prime}$ is the dimensional radious of the outer cylinder, $V_{0}$ is the dimensional reference velocity, $\sigma$ is the electric conductivity and $\mu$ is the magnetic permeability of the liquid metal.

In the assumption of the fully developed laminar forced flow all the variables $\vec{V}=\mathrm{u} \hat{e}_{r}+\mathrm{v} \hat{e}_{\theta}+\mathrm{w} \hat{e}_{z}, \vec{B}$ and $\vec{J}$ are independent of the axial coordinate $z$, except for the pressure, which is a linear function of $z$. Hence all the axial derivatives are neglected, except for the axial pressure gradient $p_{a, z}=\partial P(z) / \partial z$.

The Cartesian coordinates are transformed to a toroidal-poloidal coordinate system $(r, \theta, z)$ using the non-dimensional transformations $x^{\prime}=\left(R_{c}+r \cos \theta\right) \cos \cos \left(z / R_{c}\right)$ '

$y^{\prime}=r \sin \theta$

$z^{\prime}=\left(R_{c}+r \cos \theta\right) \sin \sin \left(z / R_{c}\right)$

where $0 \leq z \leq 2 \pi R_{c}, 0 \leq \theta \leq 2 \pi$

Introducing the term $I=1 /(1+\kappa r \cos \theta)$, where $\kappa=1 / R_{c}$ is the curvature ratio, the governing equations of the fully developed MHD and thermal flow are described below

Continuity equation

$\frac{\partial u}{\partial r}+\frac{u}{r}+\frac{1}{r} \frac{\partial v}{\partial \theta}+\kappa(u \cos \theta-v \sin \theta)=0$

Momentum equations at $r$-direction,

$\frac{\partial u}{\partial t}+u \frac{\partial u}{\partial r}+\frac{v}{r} \frac{\partial u}{\partial \theta}-\frac{v^{2}}{r}-\kappa w^{2} \cos \theta=-\frac{\partial P}{\partial r}$

$+\frac{1}{\operatorname{Re}}\left[\begin{array}{c}\frac{\partial^{2} u}{\partial r^{2}}+\left(\frac{1}{r}+\kappa \cos \theta\right) \frac{\partial u}{\partial r}+\frac{1}{r^{2}} \frac{\partial^{2} u}{\partial \theta^{2}}-\kappa \frac{\sin \theta}{r}\left(\frac{\partial u}{\partial \theta}-v\right) \\ -\frac{2}{r^{2}} \frac{\partial v}{\partial \theta}-\frac{u}{r^{2}}+(\kappa)^{2}(v \sin \theta-u \cos \theta) \cos \theta\end{array}\right]$

$$
+\frac{G r}{\operatorname{Re}^{2}} T \sin \theta+\frac{H a^{2}}{\operatorname{Re}}\left[J_{\theta} B_{i z}-J_{z} B_{o \theta}\right]
$$

at $\theta$-direction,

$$
\begin{gathered}
\frac{\partial v}{\partial t}+u \frac{\partial v}{\partial r}+\frac{v}{r} \frac{\partial v}{\partial \theta}+\frac{u v}{r}+\kappa w^{2} \sin \theta=-\frac{1}{r} \frac{\partial P}{\partial \theta} \\
+\frac{1}{\operatorname{Re}}\left[\begin{array}{c}
\frac{\partial^{2} v}{\partial r^{2}}+\left(\frac{1}{r}+\kappa \cos \theta\right) \frac{\partial v}{\partial r}+\frac{1}{r^{2}} \frac{\partial^{2} v}{\partial \theta^{2}}-\kappa \frac{\sin \theta}{r}\left(\frac{\partial v}{\partial \theta}+u\right) \\
+\frac{2}{r^{2}} \frac{\partial u}{\partial \theta}-\frac{v}{r^{2}}-(\kappa)^{2}(v \sin \theta-u \cos \theta) \sin \theta
\end{array}\right] \\
+\frac{G r}{\operatorname{Re}^{2}} T \cos \theta+\frac{H a^{2}}{\operatorname{Re}}\left[J_{z} B_{o r}-J_{r} B_{i z}\right]
\end{gathered}
$$

and at $\mathrm{z}$-direction

$\frac{\partial w}{\partial t}+u \frac{\partial w}{\partial r}+\frac{v}{r} \frac{\partial w}{\partial \theta}+\kappa \boldsymbol{L} w(u \cos \theta-v \sin \theta)=-I p_{a, z}$

$+\frac{1}{\operatorname{Re}}\left[\frac{\partial^{2} w}{\partial r^{2}}+\left(\frac{1}{r}+\kappa \cos \theta\right) \frac{\partial w}{\partial r}+\frac{1}{r^{2}} \frac{\partial^{2} w}{\partial \theta^{2}}-\kappa \frac{\sin \theta}{r} \frac{\partial w}{\partial \theta}\right.$

$$
\left.-(k I)^{2}\right]+\frac{H a^{2}}{\operatorname{Re}}\left[J_{r} B_{o \theta}-J_{\theta} B_{o r}\right]
$$

Energy equation

$\frac{\partial T}{\partial t}+u \frac{\partial T}{\partial r}+\frac{v}{r} \frac{\partial T}{\partial \theta}=\frac{1}{\operatorname{RePr}}\left[\begin{array}{c}\frac{\partial^{2} T}{\partial r^{2}}+\left(\frac{1}{r}+\kappa \cos \theta\right) \frac{\partial T}{\partial r}-\kappa \frac{\sin \theta}{r}\left(\frac{\partial T}{\partial \theta}\right) \\ +\frac{1}{r^{2}} \frac{\partial^{2} T}{\partial \theta^{2}}\end{array}\right]$

Magnetic field induction equations

$r$-direction 
Citation: Bakalis PA, Hatzikonstantinou PM (2016) Effect of the Magnetic Field on the Flow of a Liquid Metal and Heat Transfer in a Curved Cylindrical Annular Duct with Uniform Wall Temperatures. Int J Mech Syst Eng 2: 120. https://doi.org/10.15344/2455-7412/2016/120

Page 3 of 11

$$
\begin{gathered}
\frac{\partial}{R_{m}}\left[\begin{array}{c}
\frac{\partial B_{i r}}{\partial t}+u \frac{\partial B_{i r}}{\partial r}+\frac{v}{r} \frac{\partial B_{i r}}{\partial \theta} \\
-\frac{2}{r^{2}}+\left(\frac{1}{r}+\kappa \cos \theta\right) \frac{\partial B_{i r}}{\partial \theta}+\frac{1}{r^{2}} \frac{\partial^{2} B_{i r}}{\partial \theta^{2}}-\kappa\left[\frac{B_{i r}}{r^{2}}+(\kappa)\right)^{2}\left(B_{i \theta} \sin \theta-B_{i r} \cos \theta\right) \cos \theta \\
+B_{i r} \frac{\partial v}{\partial r}+\frac{B_{i \theta}}{r} \frac{\partial v}{\partial \theta}+B_{o r} \frac{\partial v}{\partial r}+\frac{B_{o \theta}}{r} \frac{\partial v}{\partial \theta}-u \frac{\partial B_{o \theta}}{\partial r}-\frac{v}{r} \frac{\partial B_{o \theta}}{\partial \theta}
\end{array}\right]
\end{gathered}
$$

$\theta$-direction

$$
\begin{gathered}
\frac{1}{R_{m}}\left[\begin{array}{c}
\frac{\partial B_{i \theta}}{\partial t}+u \frac{\partial B_{i \theta}}{\partial r}+\frac{v}{r} \frac{\partial B_{i \theta}}{\partial \theta}+\left(\frac{1}{r}+\kappa \cos \theta\right) \frac{\partial B_{i \theta}}{\partial r}+\frac{1}{r^{2}} \frac{\partial^{2} B_{i r}}{\partial \theta^{2}}-\kappa \mathrm{L} \frac{\sin \theta}{r}\left(\frac{\partial B_{i \theta}}{\partial \theta}+B_{i r}\right) \\
\left.+\frac{2}{r^{2}} \frac{\partial B_{i r}}{\partial \theta}-\frac{B_{i \theta}}{r^{2}}-(\kappa)\right)^{2}\left(B_{i \theta} \sin \theta-B_{i r} \cos \theta\right) \sin \theta \\
+B_{i r} \frac{\partial u}{\partial r}+\frac{B_{i \theta}}{r} \frac{\partial u}{\partial \theta}+B_{o r} \frac{\partial u}{\partial r}+\frac{B_{o \theta}}{r} \frac{\partial u}{\partial \theta}-u \frac{\partial B_{o r}}{\partial r}-\frac{v}{r} \frac{\partial B_{o r}}{\partial \theta}
\end{array}\right]
\end{gathered}
$$

$Z$-direction

$\frac{\partial B_{i z}}{\partial t}+u \frac{\partial B_{i z}}{\partial r}+\frac{v}{r} \frac{\partial B_{i z}}{\partial \theta}+w\left(B_{i r} I \cos \theta-B_{i \theta} I \sin \theta\right)$

$=\frac{1}{R_{m}}\left[\frac{\partial^{2} B_{i z}}{\partial r^{2}}+\left(\frac{1}{r}+\kappa \cos \theta\right) \frac{\partial B_{i z}}{\partial r}+\frac{1}{r^{2}} \frac{\partial^{2} B_{i z}}{\partial \theta^{2}}\right.$

$\left.-\kappa \frac{\sin \theta}{r} \frac{\partial B_{i z}}{\partial \theta}-(\kappa)^{2} B_{i z}\right]+B_{i r} \frac{\partial w}{\partial r}+\frac{B_{i \theta}}{r} \frac{\partial w}{\partial \theta}$

$+B_{o r} \frac{\partial w}{\partial r}+\frac{B_{o \theta}}{r} \frac{\partial w}{\partial \theta}-\kappa B_{i z}(v \sin \theta-u \cos \theta)$

$+\kappa \mathrm{d} w\left(B_{o \theta} \sin \theta-B_{o r} \cos \theta\right)$

The components of the electric current density are computed from the Ampère's law

$J_{r}=\frac{1}{R_{m}}\left[\frac{1}{r} \frac{\partial B_{i z}}{\partial \theta}-\kappa B_{i z} \sin \theta\right]$,

$J_{\theta}=-\frac{1}{R_{m}}\left[\frac{\partial B_{i z}}{\partial r}+\kappa B_{i z} \cos \theta\right], J_{z}=\frac{1}{R_{m}}\left[\frac{\partial B_{i \theta}}{\partial r}+\frac{B_{i \theta}}{r}-\frac{\partial B_{i r}}{\partial \theta}\right]$

The dimensionless radii of the inner and outer wall are respectively $R_{o}=1$ and $R_{i}=0.5$. No slip conditions are assumed for the walls, so that $u=v=w=0$ for $r=R_{i}$ and $r=R_{0}$. Due to the electrically insulating walls the induced axial magnetic field at the walls with $r=R_{i}$ and $r=R_{o}$ is $B_{i z}$ $\left(R_{i, \theta}\right)=0$ and $B_{i z}\left(R_{o, \theta}\right)=0$. The conditions for the boundary conditions for $T$ are $T_{o}=1$ for $r=R_{o}$ and $T_{i}=0$ for $r=R_{i}$.

Other quantities of interest are the shear stress at the wall, caused by the liquid metal moving past it, which will be studied using the wall shear stress coefficient and the Nusselt number which is used to study the heat transfer mechanism.

The dimensional shear stress $\tau_{\mathrm{rz}}^{\prime}$ and the resultant dimensionless local wall shear stress $c_{f}$ are given by

$\tau_{r z}^{\prime}=v \rho \frac{\partial w^{\prime}}{\partial r^{\prime}}=v \rho \frac{V_{0}}{R_{o}^{\prime}} \frac{\partial w}{\partial r} \Rightarrow c_{f}=\frac{\operatorname{Re} \tau_{r z}^{\prime}}{\rho V_{0}^{2}}=\frac{\partial w}{\partial r}$

We define as $c_{f i}=\left.\partial w \partial r\right|_{r=R i}$ the local shear stress for the inner wall and as $c_{f o}=-\partial w /\left.\partial r\right|_{r=R o}$ the local shear stress for the outer wall of the cylindrical pipe. The azimuthal wall shear stresses for each wall is then given by $\bar{c}_{f j}=\frac{1}{2 \pi} \int_{0}^{2 \pi} c_{f j}(\theta) d(\theta)$, where $j=i$ or $o$

The local Nusselt numbers are given for each wall by the following reactions

$N u_{i}=\left.\frac{\partial T}{\partial r}\right|_{r=R_{i}}$ and $N u_{o}=-\left.\frac{\partial T}{\partial r}\right|_{r=R_{o}}$

The average Nusselt numbers for each wall are then given by

$\overline{N u}_{j}=\frac{1}{2 \pi} \int_{0}^{2 \pi} N u_{j}(\theta) d \theta$, where $j=i$ or $o$

\section{Computational Methodology}

\section{CVP methodology}

The equations (10)-(18) that govern the MHD and thermal flow were solved using a methodology involving the enhanced CVP numerical variational method (Papadopoulos and Hatzikonstantinou, Bakalis and Hatzikonstantinou) for the coupling of the continuity and the Navier-Stokes equation and the coupling of the divergence free condition of the magnetic field with the magnetic field induction equation in the b-formulation. The CVP method characteristics (extreme accuracy, robustness, easy convergence and easy implementation to complex geometries) make it unique for MHD applications.

In this method the partial differential equations are discretized and solved numerically using a pseudo-transient marching algorithm, calculating the axial velocity $\mathrm{w}$ and the transverse velocity components $\overrightarrow{\mathrm{U}}=(\mathrm{u}, \mathrm{v})$ at the $n+1$ time level from their corresponding values at the $\mathrm{n}$ time level. The non-linear terms are linearized by lagging the coefficients.

The flow is considered fully developed. Therefore, the first and second order axial derivatives are equal to zero except for the axial pressure gradient $p_{a, z}$. For this case the pressure can be splitted into two terms $P=P_{a}(z)+p(r, \theta)$.

An axisymmetric parabolic profile is used as an initial guess for the velocity at time level $n=1$. Then, at each time step an estimation of the axial velocity $w^{(n+1)}$ and the transverse velocity components $\vec{U}^{*}=\left(u^{*}, v^{*}\right)$, are calculated from equation (2) for a guessed distribution of the pressure

$$
\begin{aligned}
& \frac{\partial w^{n+1}}{\partial t}+\left(\vec{U}^{n} \cdot \vec{\nabla}\right) w^{n+1}=-\frac{\partial p_{a}^{n}}{\partial z}+\frac{1}{\operatorname{Re}} \nabla^{2} w^{n+1}+F_{L}^{n}(z) \\
& \frac{\partial \vec{U}^{*}}{\partial t}+\left(\vec{U}^{n} \vec{\nabla}\right) \vec{U}^{*}=-\vec{\nabla} p^{n}+\frac{1}{\operatorname{Re}} \nabla^{2} \vec{U}^{*}+\frac{G r}{\operatorname{Re}^{2}} \vec{T}_{b}+\vec{F}_{L}^{n}(r, \theta)
\end{aligned}
$$

Where $\vec{F}_{L}(r, \theta, z)=H a^{2} / \operatorname{Re}(\vec{J} \times \vec{B})$ is the Lorenz force.

The estimated velocity field does not, in general, satisfy the continuity equation. Hence, introducing the corrections of the transverse components of the velocity $\vec{U}=(\delta u, \delta v)$ and the pressure correction $\delta \mathrm{p}(r, \theta)$ we have

$\vec{U}^{n+1}=\vec{U}^{*}+\delta \vec{U}, p^{n+1}=p^{n}+\delta p$

The next step is to compute the transverse velocities correction field. From equation (2) we have

$$
\vec{\nabla}\left(\vec{U}^{*}+\delta \vec{U}\right)=0 \Rightarrow \vec{\nabla} \cdot \delta \vec{U}=-\vec{\nabla} \cdot \vec{U}^{*} \equiv G
$$

Hence, using the irrotational form of the CVP method, at each time step, the corrections $\overrightarrow{\delta U}=(\delta u, \delta v)$ can be calculated from the following Poisson equation 
Citation: Bakalis PA, Hatzikonstantinou PM (2016) Effect of the Magnetic Field on the Flow of a Liquid Metal and Heat Transfer in a Curved Cylindrical Annular Duct with Uniform Wall Temperatures. Int J Mech Syst Eng 2: 120. https://doi.org/10.15344/2455-7412/2016/120

Page 4 of 11

$\overrightarrow{\delta U}=\vec{\nabla} G$

where $(\delta U)=0$ at the walls.

Substituting the transverse velocities (25) into equation (1) at each time level $n+1$ and by subtracting from this equation (24) we obtain

$\vec{\nabla} \delta p=-\frac{\partial \overrightarrow{\delta U}}{\partial t}-\left(\vec{U}^{n} \vec{\nabla}\right) \overrightarrow{\delta U}+\frac{1}{\mathrm{Re}} \overrightarrow{\delta U}$

where with $\vec{\nabla} \delta p \equiv \vec{f}\left(f_{r}, f_{\theta}\right)$ with $f_{r}=\partial \delta p / \partial r, f_{\theta}=\partial \delta p / r \partial \theta$.

Evaluating the value of $f$ from equation (28), the pressure correction is obtained by solving the following Poisson equation

$\delta p=\vec{\nabla} \cdot \vec{f}-G+\vec{\nabla} \overrightarrow{\delta U}$

The values of $u^{(n+1)}, v^{(n+1)}$ and $p^{(n+1)}$ can now be determined from equation (25).

During the iterative procedure the axial pressure gradient $\mathrm{p}_{\mathrm{az}}=\partial \mathrm{p} \partial \mathrm{z}$ is considered as uniform over the cross-section and is updated by the mass conservation equation so that the average axial velocity will be equal to $\bar{w}=1$. To do so we use and enhanced methodology than the one described in [23] which is determined by:

$p_{a, z}^{n+1}=\frac{{ }_{n+1}-1.0}{c \Delta t}+p_{a, z}^{n}$

where $\bar{w}_{n+1}=1 / A_{0} \int_{A_{0}} w^{n+1} d A_{0}$ and $c$ is a relaxation factor.

\section{Induced magnetic field formulation (b-formulation)}

Using the b-formulation the full set of the MHD equations is solved. The electric current density is calculated from the Ampere's law, thus the divergence free condition for the electric current density is always satisfied, since it occurs that $\vec{\nabla} \vec{J}=1 / R \vec{\nabla} \cdot\left(\vec{\nabla} \times \vec{B}_{i}\right)=0$. The b-formulationinvolves all the terms of the induced magnetic field $\vec{B}_{i}^{*}$ of the flow, without the assumptions and simplifications of the other formulations, but it requires a greater effort of computational cost.

A variational procedure is developed consistent with the CVP methodology in order to ensure that the divergence-free condition of the magnetic field is satisfied. Solving equations (15)-(17), the induced magnetic field $\vec{B}_{i}^{*}$ that occurs at each $n+1$ step, does not in general satisfy the divergence-free condition. Indeed,

$\nabla \vec{B}_{i}^{*}=\frac{1}{r} \frac{\partial\left(r \vec{B}_{i r}^{*}\right)}{\partial r}+\frac{1}{r} \frac{\partial \vec{B}_{i \theta}^{*}}{\partial \theta}=-H \neq 0$

Introducing the corrections $\delta \vec{B}_{i}=\left(\delta B_{i r}, \delta B_{i \theta}, 0\right)$ we have

$\vec{B}_{i}^{n+1}=\vec{B}_{i}^{*}+\delta \vec{B}_{i}$

From the divergence-free condition we obtain

$\vec{\nabla} B_{i}^{n+1}=\vec{\nabla}\left(\vec{B}_{i}^{*}+\delta \vec{B}_{i}\right)=0 \Rightarrow \vec{\nabla} \cdot \delta \vec{B}_{i}=-\vec{\nabla} \cdot \vec{B}_{i}^{*}=H$

Using the vector identity $\nabla^{2} \delta \vec{B}=\vec{\nabla}(\vec{\nabla} \cdot \delta \vec{B})-\vec{\nabla} \times(\vec{\nabla} \times \delta \vec{B})$ and considering the term $\vec{\nabla} \times\left(\vec{\nabla} \times \delta \vec{B}_{i}\right)$ as negligible, the corrections $\delta \vec{B}_{i}$ at each time step can be calculated by the Poisson's equation

$\nabla^{2} \delta \vec{B}=\vec{\nabla} \cdot H$

with $\delta \vec{B}_{i}=0$ on the walls. Subsequently, the values $\vec{B}_{i}^{n+1}$ of are determined from equation (31).

Table 1 shows the effect of the corrections of the induced magnetic field $\delta \vec{B}_{i}$ of equation (33) on the mean value of the absolute of the divergence of the induced magnetic field $\left|\overrightarrow{\vec{\nabla} \cdot \vec{B}_{\imath}}\right|$ for the b-formulation.
The values of $|\vec{\nabla} \cdot \vec{B}$,$| that occur using the methodology of paragraph 3.2$ are by three orders of magnitude smaller than the corresponding values of $|\overrightarrow{\bar{\nabla} \cdot \vec{B}}|$ without using a correction procedure. Thus, the method for the b-formulation significantly improves the numerical satisfaction of the divergence free condition for the induced magnetic field.

Numerical results providing the effectiveness of our methodology are presented in the next paragraph. This methodology can be incorporated with any other computational methodology for the accurate determination of the induced magnetic field in close space regions.

\begin{tabular}{|l|l|l|}
\hline $\begin{array}{l}\text { Hartmann } \\
\text { number }\end{array}$ & $\begin{array}{l}\left|\vec{\nabla} \cdot \vec{B}_{l}\right| \text { using the } \\
\text { corrections } \delta \vec{B}_{i}\end{array}$ & \multicolumn{1}{|c|}{$\begin{array}{c}\left|\vec{\nabla} \cdot \vec{B}_{l}\right| \text { without } \\
\text { correction }\end{array}$} \\
\hline $\mathrm{Ha}=100$ & $5.24 \times 10^{-10}$ & $6.88 \times 10^{-7}$ \\
\hline $\mathrm{Ha}=500$ & $4.98 \times 10^{-11}$ & $5.73 \times 10^{-8}$ \\
\hline $\mathrm{Ha}=1000$ & $2.29 \times 10^{-11}$ & $3.63 \times 10^{-8}$ \\
\hline $\mathrm{Ha}=2000$ & $1.88 \times 10^{-11}$ & $2.56 \times 10^{-8}$ \\
\hline
\end{tabular}

\section{Computational algorithm and grid}

The iterative algorithm begins with an estimation of the velocity and the axial pressure gradient. The velocity components and the pressure are evaluated from the CVP algorithm and with these estimated values the axial component of the induced magnetic field and electric current density are computed using the b-formulation and thereafter the axial pressure gradient is updated. The iteration is repeated until the following convergence criterion for the velocity components is achieved for two sequential time steps,

$$
\begin{aligned}
& \left|\frac{\psi_{i, j}^{n+1}-\psi_{i, j}^{n}}{\psi_{i, j}^{n+1}}\right|<\varepsilon=10^{-5} \\
& \text { where } \psi_{i, j}=u_{(i, j)}, v_{(i, j)}, w_{(i, j)} \text { and } n \text { represents the iteration number. }
\end{aligned}
$$

Non-uniform stretched meshes [17,23] are used in order to compute accurately the MHD boundary layers near the walls, and particularly the Hartmann, and side layers, which are respectively vertical and parallel to the magnetic field. Thus, special attention should be paid for the regions near the inner and the outer walls, where thin velocity and electric current layers are formulated due to the effect of the magnetic field, as well as in the right half region of the annulus, where the maximum velocity is formulated due to the effect of the centrifugal and Lorentz forces.

To refine the mesh near the walls, the following transformation for the r-coordinate is used [19]

$$
\begin{aligned}
& r_{i}=R_{i}+\left(R_{o}-R_{i}\right) \frac{(2 a+b)\left(\frac{b+1}{b-1}\right)^{\frac{\bar{r}_{-}-a}{1-a}}+2 a-b}{(2 a+1)\left[\left(\frac{b+1}{b-1}\right)^{\frac{r_{-}-a}{1-a}}+1\right]} \\
& \text { where } a=0.5, b=\sqrt{\sqrt{H a} / \sqrt{H a-1}} \text { is a stretching parameter and } i= \\
& 1,2, \ldots, I .
\end{aligned}
$$

With this formula, a uniform mesh ${ }^{-}$can be transformed into a refined mesh $r_{i}$. Since $a=0.5$, the mesh will be refined equally near $R_{i}$ and $R_{0}$. The stretching parameter $b$ will be related with the Hartmann number, because as the Hartmann number increases, the layers near the walls decrease in thickness. 
Citation: Bakalis PA, Hatzikonstantinou PM (2016) Effect of the Magnetic Field on the Flow of a Liquid Metal and Heat Transfer in a Curved Cylindrical Annular Duct with Uniform Wall Temperatures. Int J Mech Syst Eng 2: 120. https://doi.org/10.15344/2455-7412/2016/120

Page 5 of 11

The following transformation is used in $\theta$-direction twice, in the region between 0 to $\pi$ and in the region between $\pi$ to $2 \pi$, in order to refine the mesh in the left half region at the left side of the annulus

$\theta_{j}=s_{c}\left[1+\frac{\sinh \sinh \left[\tau\left(\bar{\theta}_{j}-\gamma\right)\right]}{\sinh [\tau \gamma]}\right]$

$\gamma=\frac{1}{2 \tau} \ln \left[\frac{1+\left(\mathrm{e}^{\hat{o}}-1\right)\left(s_{c} / \pi\right)}{1+\left(\mathrm{e}^{-\hat{o}}-1\right)\left(s_{c} / \pi\right)}\right]$

where $s_{c}$ is the refinement point, $\tau$ is the stretching parameter and $\bar{\theta}_{j}$ corresponds to a uniform $\theta$ mesh.

The following stretching parameters were used for the relations (34) and (35) are

$a=0.5, b=\sqrt{\sqrt{H a} / \sqrt{H a-1}}, s_{c}=0.25$ and $\tau=4$.

The effect of the grid on the numerical results for various Hartmann numbers is presented in our work (Bakalis \& Hatzikonstantinou, 2015).
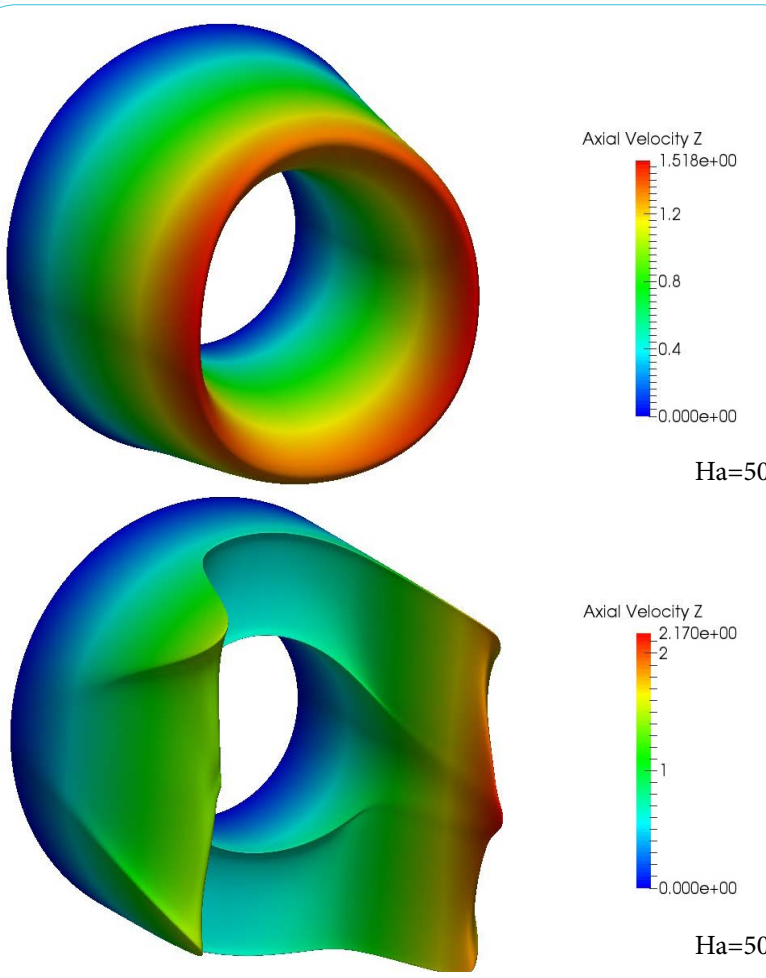

$\mathrm{Ha}=50$

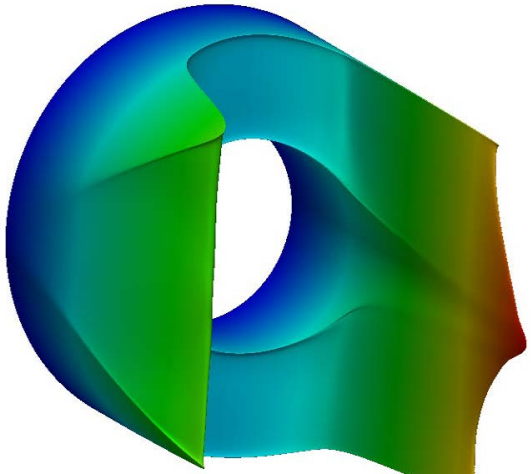

Axial Velocity

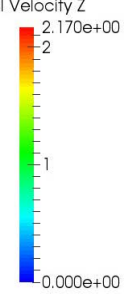

$\mathrm{Ha}=50$

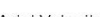

Axial Velocity 2
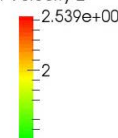

Figure 2: 3D plot of the axial velocity for various Hartmann numbers for $R e=2000, G r=0, \kappa=0.2$. Left is the inner region and right is the outer region of the curved duct.

\section{Result \& Discussion}

The magnetohydrodynamic and thermal flow of a liquid metal in a curved annular pipe is studied in the present work for $\mathrm{Ha}=0-2000$, $R e=100-2000, G r=0-10^{6}, P r=0.0612, R m=0.001$, and $k=0-0.2$.

The results are illustrated in order to demonstrate the effect of the curvature and the magnetic field on the velocity distribution. The decrease of curvature $\kappa$ yields to the decrease of the axial velocity and the reduction of the centrifugal forces. The effect of curvature on MHD flow is presented in our work [18].

The 3D contour plot of Fig. 2 clearly represents the effect of the magnetic field on the axial velocity distribution, for various Hartmann numbers and for $R e=2000, G r=0$ and $\kappa=0.2$. As it can be observed as the Hartmann number increases side velocity jets are formulated in the left and right regions of the ring-shaped cross section, in a direction parallel to the external magnetic field and the magnitude of the axial velocity $\mathrm{w}$ is strongly suppressed at the regions of the Hartmann layers $\left(\theta=90^{\circ}\right.$ and $\left.270^{\circ}\right)$. These results indicate that as the external magnetic field increases, the liquid metal tends to move mainly by the side regions parallel to the direction of the external magnetic field and tends to be nonmoving at the top and bottom wall regions normal to the external magnetic field.

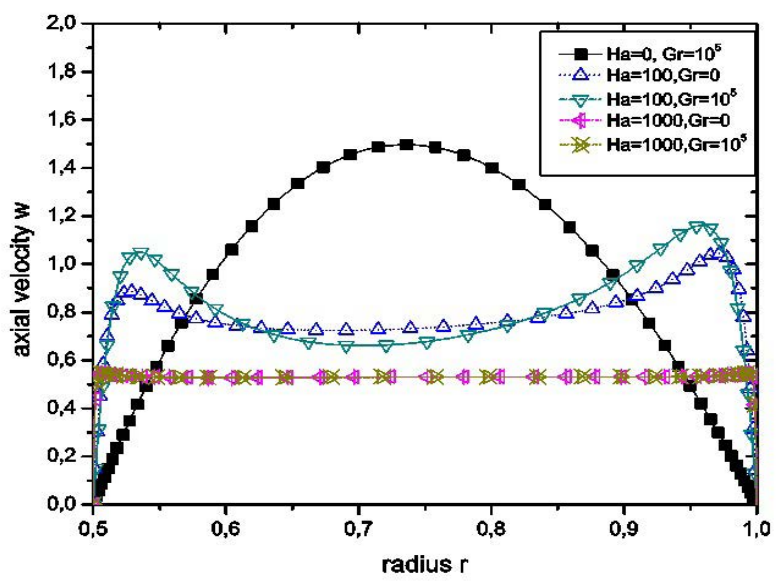

Figure 3: Axial velocity profile along the $r$-axis at angle $r=90^{\circ}$ for $\mathrm{Ha}=0$ $100,1000, G r=0,10^{5}$ and $R e=1000, \kappa=0.2$.

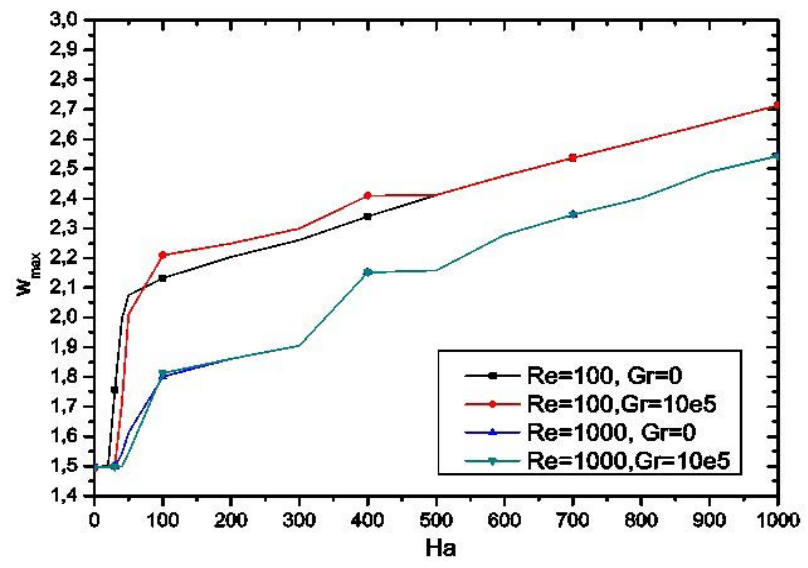

Figure 4: Maximum value of the axial velocity $w_{\max }$ at various Hartmann numbers for $R e=100,1000$ and $G r=0,10^{5}, \kappa=0.2$. 
Citation: Bakalis PA, Hatzikonstantinou PM (2016) Effect of the Magnetic Field on the Flow of a Liquid Metal and Heat Transfer in a Curved Cylindrical Annular Duct with Uniform Wall Temperatures. Int J Mech Syst Eng 2: 120. https://doi.org/10.15344/2455-7412/2016/120

Page 6 of 11

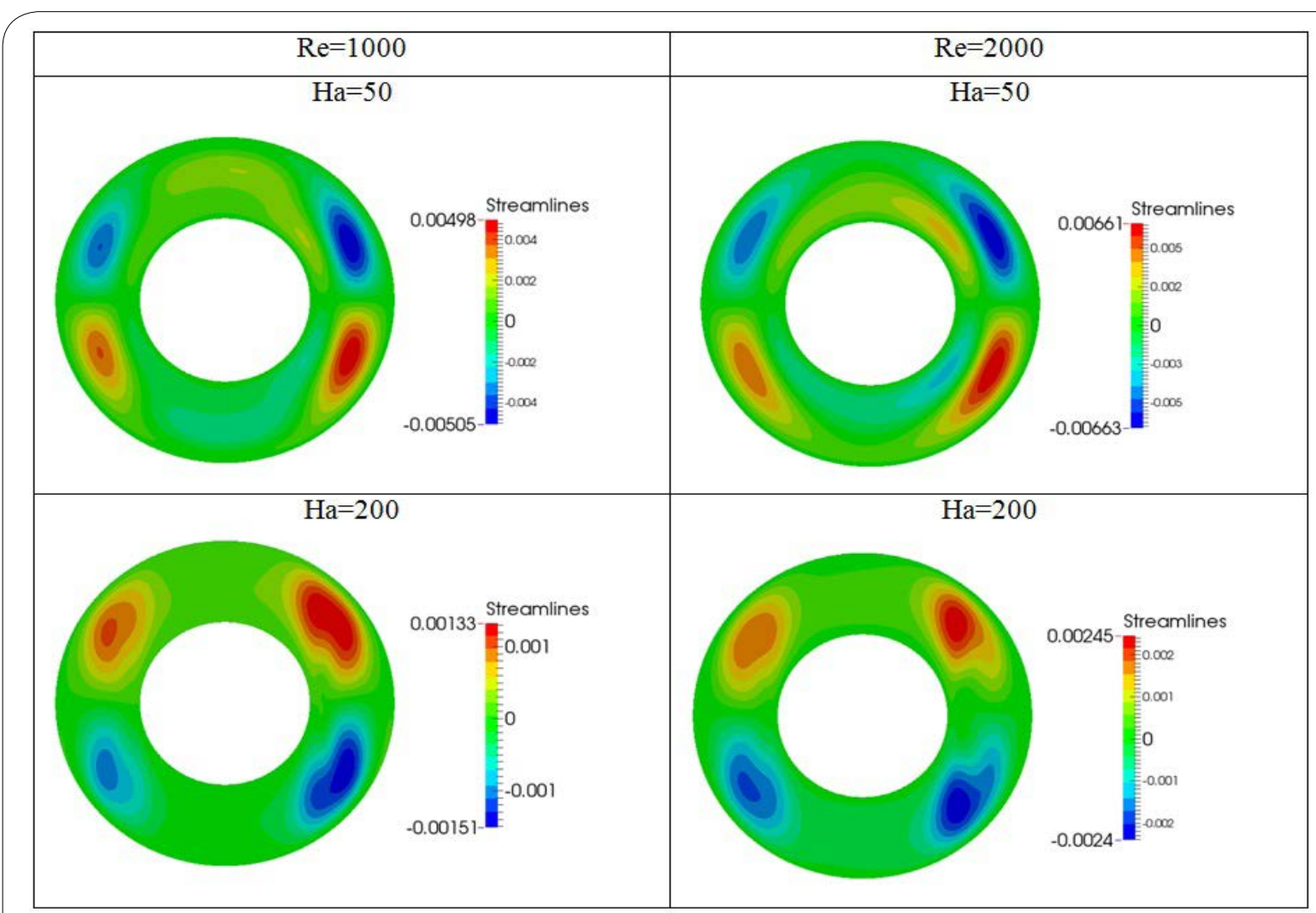

Figure 5: Contour plot of the stream function of the transverse components of the velocity for various Hartmann numbers, for $G r=0$ and $R e=1000,2000, \kappa=0.2$.

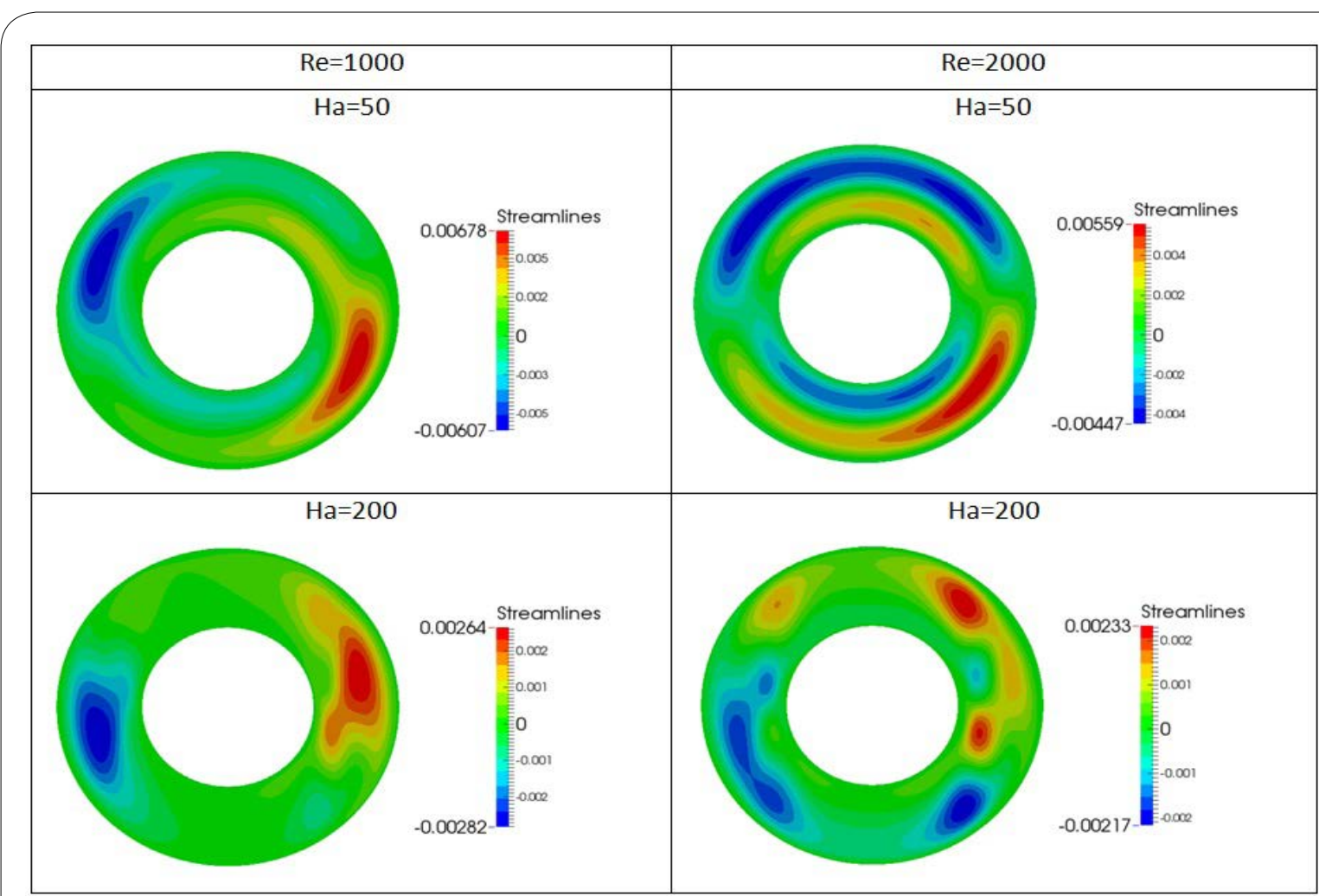

Figure 6: Contour plot of the stream function of the transverse components of the velocity for various Hartmann numbers, for $\mathrm{Gr}=10^{5}$ and $R e=1000,2000, \kappa=0.2$. 
Citation: Bakalis PA, Hatzikonstantinou PM (2016) Effect of the Magnetic Field on the Flow of a Liquid Metal and Heat Transfer in a Curved Cylindrical Annular Duct with Uniform Wall Temperatures. Int J Mech Syst Eng 2: 120. https://doi.org/10.15344/2455-7412/2016/120

Page 7 of 11

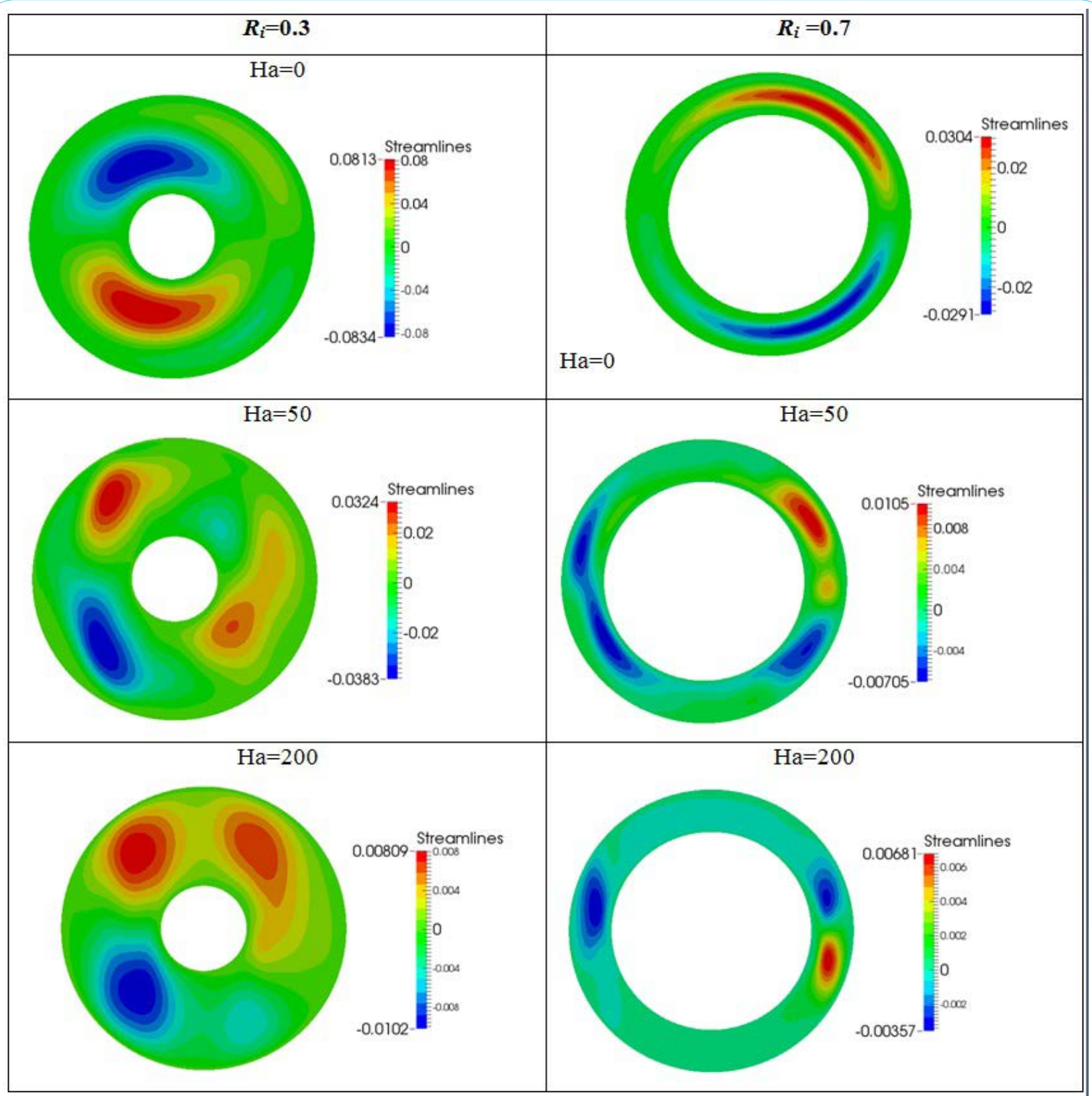

Figure 7: Contour plot of the stream function of the transverse components of the velocity for various Hartmann numbers for $R e=1000$, $G r=10^{5}$, $k=0.2$ and for different inner cylinder diameters than the standard case.

The suppression of the axial velocity at the regions of the Hartmann layers $\left(\theta=90^{\circ}\right.$ and $\left.270^{\circ}\right)$ is presented in Figure 3 with the combination of the effect of the Grashof number. It can be observed that the axial velocity profile flattens between the walls and exhibits thin Hartmann layers near the walls (vertical to $\vec{B}$ ), as a result of the direction of the Lorenz Force. The Hartmann layers decrease in thickness as the Hartmann number increases. For $\mathrm{Ha}=1000$ the profiles for the two different Grashof numbers are identical.

Figure 4 represents the variation of the maximum value of the axial velocity $w_{\max }$, which appers at angle $\theta=180^{\circ}$, in terms of the Hartmann number and for $R e=100,1000, G r=0,10^{5}$ and $\kappa=0.2$. The peak of the axial velocity increases as the Hartmann number increases, while the effect of the Grashof number is smaller.
The contour plots of the stream functions of the transverse velocities for various values of the Hartmann and Reynolds numbers are shown for $G r=0$ at Figure 5 and for $G r=10^{5}$ at Figure 6. As the magnetic field increases the transverse components of the velocity are suppressed and two pairs of vortices are redistributed. On the other hand, on Figure 6 it is shown that as the buoyancy forces increase to high values, a stirring of one pair of vortices is presented which increases further as the Reynolds number increases. However, as the Hartmann number increases to higher values, the secondary flow tends to restore the two pair of vortices. For Hartmann numbers greater than 200, the secondary flow is suppressed by orders of magnitude, regardless of the presence of the buoyancy forces.

The stream functions of the transverse components of the velocity are presented in Figure 7 for $R e=1000, G r=10^{5}, \kappa=0.2$ and for two 
Citation: Bakalis PA, Hatzikonstantinou PM (2016) Effect of the Magnetic Field on the Flow of a Liquid Metal and Heat Transfer in a Curved Cylindrical Annular Duct with Uniform Wall Temperatures. Int J Mech Syst Eng 2: 120. https://doi.org/10.15344/2455-7412/2016/120

Page 8 of 11

different inner diameters $\left(\mathrm{R}_{\mathrm{i}}=0.3\right.$ and $\left.\mathrm{R}=0.7\right)$ than the standard case It is observed that whereas for the case with inner diameter $\mathrm{R}_{i}=0.3$ the magnitude of the streamlines is higher than that of $\mathrm{R}_{i}=0.5$ and for $\mathrm{R}_{i}=0.7$ is lower than that of $\mathrm{R}_{i}=0.5$, in both cases, the effect of the external magnetic field highly suppresses the secondary flow and for $\mathrm{Ha}=200$ it has been reduced by one order of magnitude.

Table 2 represents the effect of the magnetic field and the Reynolds number on the heat transfer as it is expressed by the average Nusselt number on the inner and the outer cylinder, for Grashof number $G r=0$ and $\kappa=0.2$. It is observed that for small Hartmann numbers, as the Reynolds number increases the average Nusselt number of the inner cylinder decreases and that of the outer cylinder increases, as the secondary flow is shifted to the outer cylinder. Even though, for Hartmann numbers higher than $\mathrm{Ha}=50$ the heat transfer is uniform regardless of the value of the Reynolds number, this is due to the suppression of the secondary flow from the magnetic field.

Table 3 represents the effect of the magnetic field on the average Nusselt numbers, but this time for different values of the Grashof number and for Reynolds number $R e=1000$. For small Hartmann numbers, under the presence of the Grashof number, the heat transfer on the inner cylinder decreases, while on the outer cylinder it highly increases. This is due to the higher circulation of the secondary flow near the outer wall. For Hartmann numbers higher than $\mathrm{Ha}=100$, the heat transfer is unified, regardless of the presence of the Grashof number or its value, due to the suppression of the secondary flow.

\begin{tabular}{|c|c|c|c|}
\hline \multicolumn{4}{|c|}{$\mathrm{Nu}_{\mathrm{i}}$} \\
\hline & \multicolumn{3}{|c|}{$\mathrm{Re}$} \\
\hline $\mathrm{Ha}$ & 100 & 1000 & 2000 \\
\hline 0 & 0,46 & 0,42 & 0,38 \\
\hline 10 & 0,96 & 0,82 & 0,72 \\
\hline 20 & 1,44 & 1,22 & 1,05 \\
\hline 50 & 2,88 & 2,87 & 2,88 \\
\hline 100 & 2,88 & 2,88 & 2,88 \\
\hline 1000 & 2,88 & 2,88 & 2,88 \\
\hline
\end{tabular}

\begin{tabular}{|c|c|c|c|}
\hline \multicolumn{5}{|c|}{$N u_{o}$} \\
\hline & \multicolumn{3}{|c|}{$\operatorname{Re}$} \\
\hline $\mathrm{Ha}$ & 100 & 1000 & 2000 \\
\hline 0 & 5,17 & 5,29 & 5,41 \\
\hline 10 & 3,61 & 3,83 & 4,02 \\
\hline 20 & 2,77 & 3,02 & 3,24 \\
\hline 50 & 1,45 & 1,45 & 1,45 \\
\hline 100 & 1,45 & 1,45 & 1,46 \\
\hline 1000 & 1,45 & 1,45 & 1,45 \\
\hline
\end{tabular}

Table 2: Effect of the magnetic field and Reynolds number on the inner and outer Nusselt number for $G r=0, k=0.2$ and different values of Hartmann and Reynolds number.

In Figure 8 the contour plot of temperature is presented for various Hartmann and Grashof numbers and for $R e=1000$ and $\kappa=0.2$. It can be observed that the temperature profile is uniform at $\theta$-direction, due to the low value of the Prandtl number. Even though, as the Hartmann number increases from $\mathrm{Ha}=0$ to $\mathrm{Ha}=50$ there is a shift of the temperature profile and an increase of its values. This is due to the

\begin{tabular}{|c|c|c|c|c|}
\hline \multicolumn{5}{|c|}{$\mathrm{Nu}_{\mathrm{i}}$} \\
\hline \multicolumn{5}{|c|}{$\mathrm{Gr}$} \\
\hline $\mathrm{Ha}$ & 0 & 104 & 105 & 106 \\
\hline 0 & 0,42 & 0,22 & 0,22 & 0,23 \\
\hline 10 & 0,82 & 0,39 & 0,39 & 0,39 \\
\hline 20 & 1,22 & 0,56 & 0,56 & 0,56 \\
\hline 50 & 2,87 & 2,72 & 2,72 & 2,81 \\
\hline 100 & 2,88 & 2,87 & 2,87 & 2,98 \\
\hline 1000 & 2,88 & 2,88 & 2,88 & 2,87 \\
\hline \multicolumn{5}{|c|}{$\mathrm{Nu}_{\mathrm{o}}$} \\
\hline \multicolumn{5}{|c|}{$\mathrm{Gr}$} \\
\hline $\mathrm{Ha}$ & 0 & 104 & 105 & 106 \\
\hline 0 & 5,29 & 6,55 & 6,55 & 6,55 \\
\hline 10 & 3,83 & 5,33 & 5,33 & 5,33 \\
\hline 20 & 3,02 & 4,05 & 4,05 & 4,05 \\
\hline 50 & 1,45 & 1,55 & 1,55 & 1,6 \\
\hline 100 & 1,45 & 1,46 & 1,46 & 1,57 \\
\hline 1000 & 1,45 & 1,45 & 1,45 & 1,46 \\
\hline
\end{tabular}

Table 3: Effect of the magnetic field and buoyancy forces on the average inner and outer Nusselt numbers for $R e=1000, k=0.2$ and different values of Hartmann and Grashof number.

increase of the velocity near the outer wall. From Hartmann numbers higher than $\mathrm{Ha}=50$ the temperature profile remains intact.

The contour plots of the induced axial magnetic field are presented in Figure 9 for $\mathrm{Ha}=10,200, \mathrm{Gr}=0,10^{5}$ and $\kappa=0.2$. For $\mathrm{Ha}<100$ the induced magnetic field is distributed in two zones parallel to the Hartmann layers. However For $\mathrm{Ha}>100$ the induced magnetic field is distributed into two regions with negative values near $\theta=45^{\circ}$ and $\theta=135^{\circ}$ and two regions with positive values near $\theta=-45^{\circ}$ and $\theta=-$ $135^{\circ}$. As the Hartmann number increases the values of the induced magnetic field with and without the effect of the buoyancy forces tend to be equalized due to the suppress of the secondary flow.

Table 4 represents the effect of the magnetic field and the Reynolds number on the average friction factor of the inner and the outer cylinder for Reynolds number $\mathrm{Re}=1000$ and $\kappa=0.2$. As it can be observed, increasing the Hartmann number highly increases the friction factor on both the inner and the outer cylinder, due to the presence of the Hartmann and side layers and the increase of the axial velocity magnitude near the walls.

Table 4 represents the variation of the axial pressure gradient $p_{a, z}$ for various values of the Hartmann and Reynolds numbers, for Grashof number $G r=0$, and $\kappa=0.2$. As the Hartmann number increases the absolute value of the axial pressure gradient highly increases, due to the effect of the opposite direction of the Lorentz force on the direction of the flow.

On the other hand, the effect of the buoyancy forces on the axial pressure gradient is significantly lower as it is presented on Table 6 for Reynolds number $R e=1000$ and $\kappa=0.2$. The impact of the Grashof number on the variation of the axial pressure gradient is significantly small, thus the required pump power on this types of flows can be accurately determined by the effect of the Lorentz force exclusively. 
Citation: Bakalis PA, Hatzikonstantinou PM (2016) Effect of the Magnetic Field on the Flow of a Liquid Metal and Heat Transfer in a Curved Cylindrical Annular Duct with Uniform Wall Temperatures. Int J Mech Syst Eng 2: 120. https://doi.org/10.15344/2455-7412/2016/120

Page 9 of 11

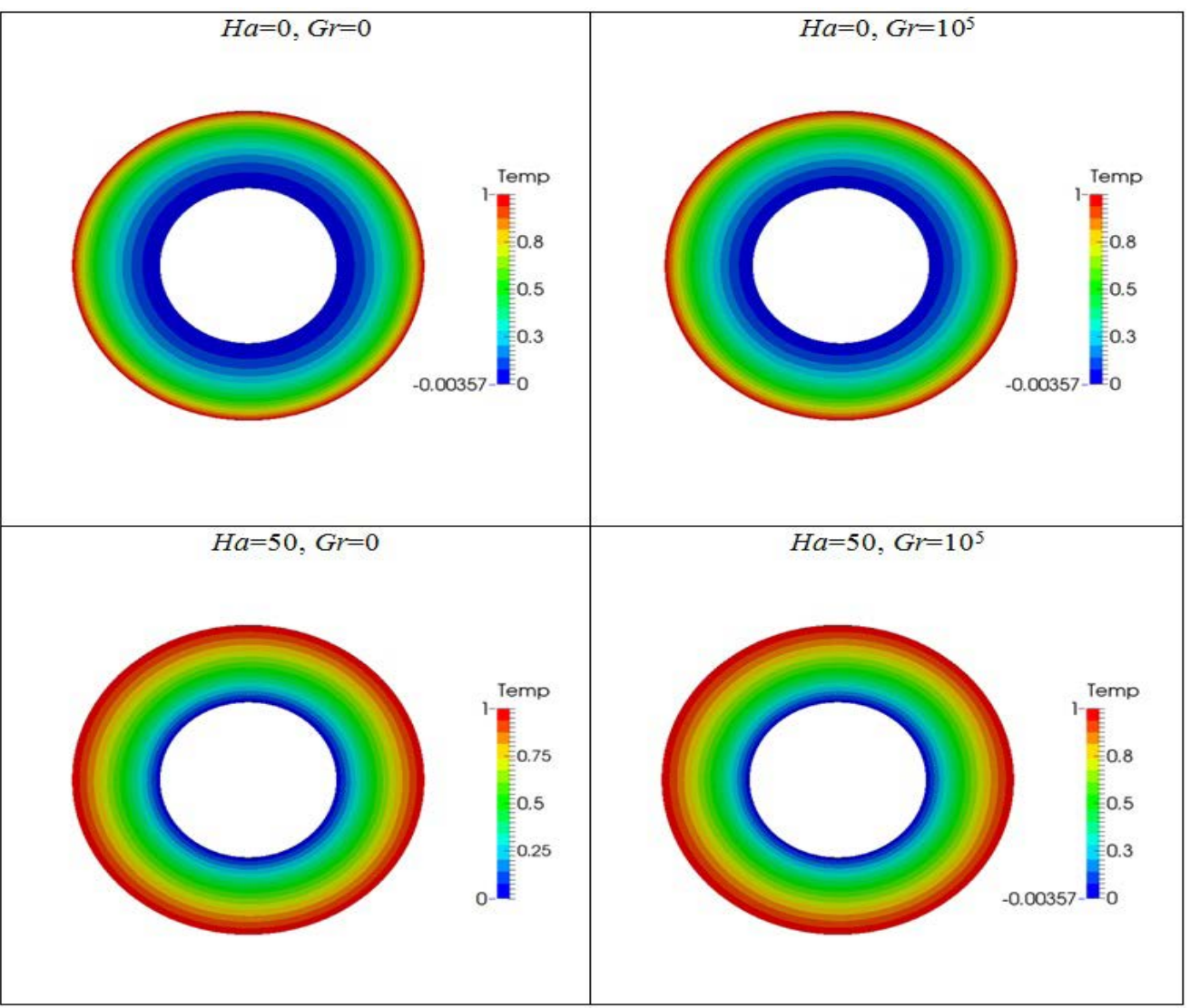

Figure 8: Contour plot of the temperature for various Hartmann and Grashof numbers and for $R e=1000, k=0.2$.

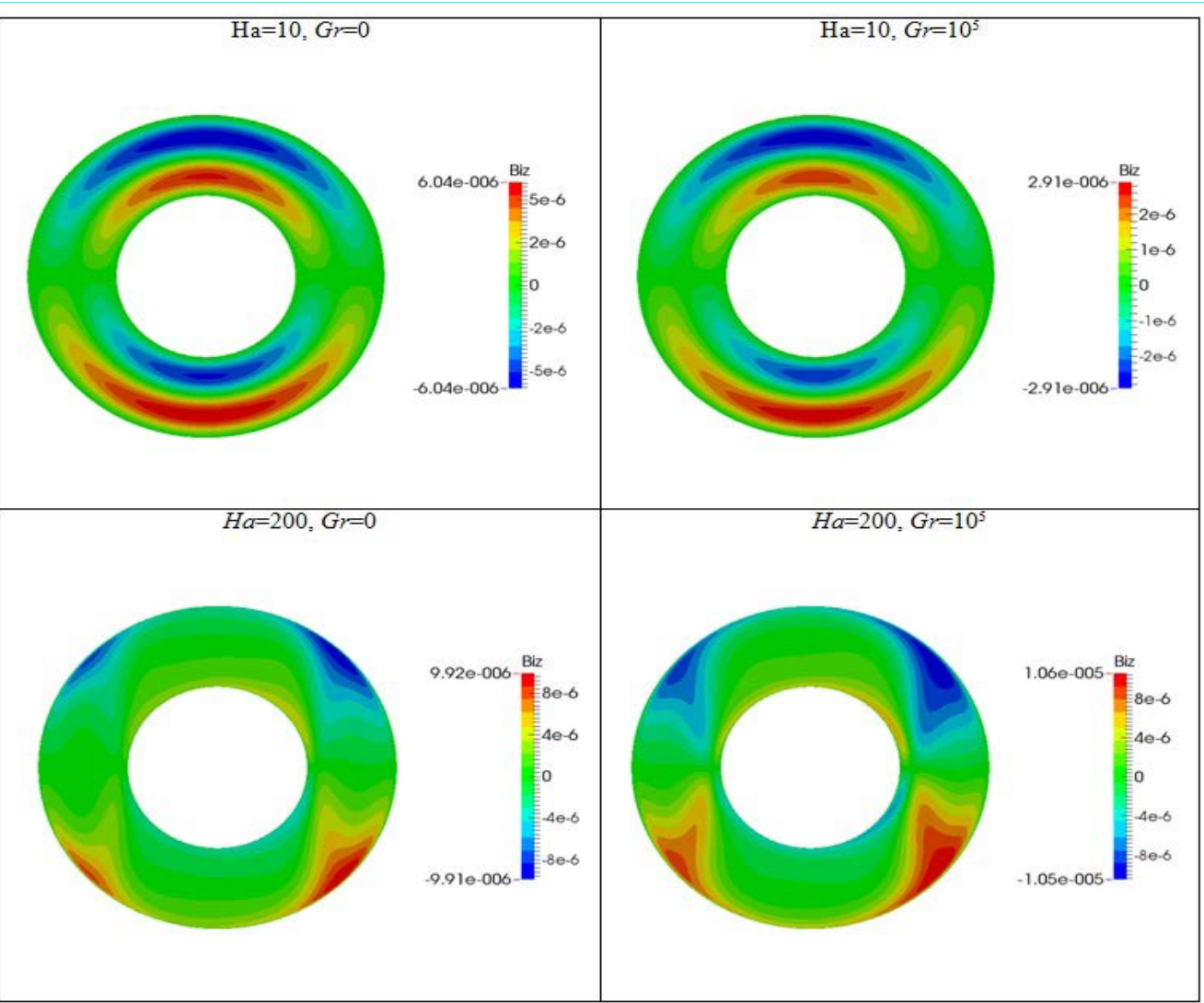

Figure 9: Contour plot of the axial induced magnetic field for various Hartmann and Grashof numbers and for $R e=1000, \kappa=0.2$. 
Citation: Bakalis PA, Hatzikonstantinou PM (2016) Effect of the Magnetic Field on the Flow of a Liquid Metal and Heat Transfer in a Curved Cylindrical Annular Duct with Uniform Wall Temperatures. Int J Mech Syst Eng 2: 120. https://doi.org/10.15344/2455-7412/2016/120

Page 10 of 11

\begin{tabular}{|c|c|c|c|}
\hline \multicolumn{5}{|c|}{$\bar{c}_{f i}$} \\
\hline & $\operatorname{Re}$ & & \\
\hline $\mathrm{Ha}$ & 100 & 1000 & 2000 \\
\hline 0 & 13,77 & 13,65 & 13,54 \\
\hline 10 & 13,78 & 13,68 & 13,60 \\
\hline 20 & 13,82 & 13,72 & 13,64 \\
\hline 50 & 34,85 & 34,85 & 32,52 \\
\hline 100 & 64,56 & 80,39 & 81,67 \\
\hline 200 & 102,25 & 131,37 & 138,51 \\
\hline 500 & 217,70 & 246,58 & 259,38 \\
\hline 1000 & 404,47 & 423,99 & 446,10 \\
\hline 2000 & 722,25 & 757,11 & 763,76 \\
\hline
\end{tabular}

\begin{tabular}{|c|c|c|c|}
\hline \multicolumn{4}{|c|}{$\bar{c}_{f o}$} \\
\hline & \multicolumn{3}{|c|}{$\mathrm{Re}$} \\
\hline $\mathrm{Ha}$ & 100 & 1000 & 2000 \\
\hline 0 & 10,86 & 10,73 & 10,61 \\
\hline 10 & 10,87 & 10,76 & 10,67 \\
\hline 20 & 10,90 & 10,80 & 10,72 \\
\hline 50 & 35,05 & 30,68 & 28,09 \\
\hline 100 & 83,79 & 82,76 & 79,67 \\
\hline 200 & 164,98 & 173,12 & 173,48 \\
\hline 500 & 335,57 & 355,98 & 339,26 \\
\hline 1000 & 635,65 & 658,30 & 657,13 \\
\hline 2000 & 1172,11 & 1213,88 & 1215,32 \\
\hline
\end{tabular}

Table 4: represents the variation of the axial pressure gradient $p_{a z}$ for various values of the Hartmann and Reynolds numbers, for Grashof number $\mathrm{Gr}=0$, and $\kappa=0.2$. As the Hartmann number increases the absolute value of the axial pressure gradient highly increases, due to the effect of the opposite direction of the Lorentz force on the direction of the flow.

\begin{tabular}{|c|c|c|c|}
\hline \multicolumn{3}{|c|}{$p_{a, z}$} \\
\hline & \multicolumn{3}{|c|}{$R e$} \\
\hline Ha & 100 & 1000 & 2000 \\
\hline 0 & $-0,48$ & $-0,05$ & $-0,02$ \\
\hline 10 & $-0,48$ & $-0,05$ & $-0,02$ \\
\hline 20 & $-0,52$ & $-0,05$ & $-0,02$ \\
\hline 50 & $-1,45$ & $-0,15$ & $-0,07$ \\
\hline 100 & $-2,59$ & $-0,31$ & $-0,17$ \\
\hline 200 & $-4,55$ & $-0,51$ & $-0,25$ \\
\hline 500 & $-10,54$ & $-1,14$ & $-0,57$ \\
\hline 1000 & $-20,49$ & $-2,11$ & $-1,08$ \\
\hline 2000 & $-39,33$ & $-4,05$ & $-2,02$ \\
\hline
\end{tabular}

Table 5: Effect of the magnetic field and Reynolds number on the axial pressure gradient for $G r=0, k=0.2$ and different values of Hartmann and Reynolds number.

\begin{tabular}{|c|c|c|c|c|}
\hline \multicolumn{5}{|c|}{$p_{a, z}$} \\
\hline & \multicolumn{5}{|c|}{$\mathrm{Gr}$} \\
\hline $\mathrm{Ha}$ & 0 & $1,00 \mathrm{E}+04$ & $1,00 \mathrm{E}+05$ & $1,00 \mathrm{E}+06$ \\
\hline 0 & $-0,05$ & $-0,06$ & $-0,07$ & $-0,07$ \\
\hline 10 & $-0,05$ & $-0,05$ & $-0,05$ & $-0,05$ \\
\hline 20 & $-0,05$ & $-0,05$ & $-0,05$ & $-0,05$ \\
\hline 50 & $-0,15$ & $-0,11$ & $-0,11$ & $-0,11$ \\
\hline 100 & $-0,31$ & $-0,33$ & $-0,33$ & $-0,33$ \\
\hline 200 & $-0,51$ & $-0,56$ & $-0,56$ & $-0,56$ \\
\hline 500 & $-1,14$ & $-1,12$ & 1,12 & 1,12 \\
\hline 1000 & $-2,11$ & $-2,11$ & $-2,11$ & $-2,11$ \\
\hline 2000 & $-4,05$ & $-4,05$ & $-4,05$ & $-4,05$ \\
\hline
\end{tabular}

Table 6: Effect of the magnetic field and buoyancy forces on the axial pressure gradient for $R e=1000, k=0.2$ and different values of Hartmann and Grashof number.

\section{Conclusions}

The effect of the magnetic field on the velocity distribution of a liquid metal flowing in a curved annular duct with temperature difference between the inner and the outer cylinder is studied in the present work.

From the results it is observed that the axial velocity is slightly affected by the secondary flow which is created by the effect of the centrifugal forces due to the curvature of the duct and the buoyancy forces due to the temperature difference between the inner and the outer wall. It is basically formulated by the magnitude of the external magnetic field, which as it increases formulates two side regions of velocity jets in the left and right half regions of the cylinder and moves the peak of the axial velocity towards the inner wall, while in the regions at the top and the bottom of the annular duct the axial velocity is highly suppressed due to the opposite direction of the Lorentz force. The axial pressure gradient which is required to maintain the same mass flow is significantly increased as the Hartmann number increases, in order to balance the opposite effect of the Lorentz force.

A secondary flow is generated due to the effect curvature of the duct and the temperature difference between the walls. This secondary flow is highly depressed as the magnitude of the external magnetic field increases and becomes negligible for Hartmann values higher than 100 200, regardless of the values of the Reynolds or Grashof number. The curvature and Grashof number were found to have a little impact on the axial pressure gradient in comparison to the effect of the Hartmann number.

\section{Competing Interestes}

The authors declare that they have no competing interestes.

\section{References}

1. Sterl A(1990) Numerical simulation of liquid-metal MHD flows in rectangular ducts. J Fluid Mech 216: 161-191.

2. Kim C, Hadid A, Abdou A (1989) Development of a computational method for the full solution of MHD flow in fusion blankets. Fusion Eng Des 8: 265270.

3. Buhler $L$ (1998) Laminar buoyant magnetohydrodynamic flow in vertical rectangular ducts. Phys Fluids 10: 223-238. 
Citation: Bakalis PA, Hatzikonstantinou PM (2016) Effect of the Magnetic Field on the Flow of a Liquid Metal and Heat Transfer in a Curved Cylindrical Annular Duct with Uniform Wall Temperatures. Int J Mech Syst Eng 2: 120. https://doi.org/10.15344/2455-7412/2016/120

Page 11 of 11

4. Arakesi H, Kotake S (1994) A self-correcting procedure for computational liquid metal magnetohydrodynamics. J Comp Phys 110: 301-309.

5. Smolentsev S, Morley N, Abdou M (2005) Code development for analysis of MHD pressure drop reduction in a liquid metal blanket using insulation technique based on a fully developed flow model. Fusion Eng Des 73: 8393.

6. Meir AG, Schmidt PG (1994) A velocity-current formulation for stationary MHD flows. Appl. Math Comp 65: 95-109.

7. Smolentsev S, Morley NB, Abdou M, Munipalli R, Moreau R (2006) Current approaches to modeling MHD flows in the dual coolant lead lithium blanket. Magnetohydrodyn 42: 225-236.

8. Smolentsev S, Wong C, Malang S, Dagher M, Abdou M (2010) MHD considerations for the DCLL inboard blanket and access ducts. Fusion Eng Des 85: 1007-1011.

9. Tabeling P, Chabrerie JP (1981) Magnetohydrodynamic secondary flows at high Hartmann numbers. J Fluid Mech 103: 225-239.

10. Moresco P, Alboussière $T$ (2004) Experimental study of the instability of the Hartmann layer. J Fluid Mech 504: 167-181.

11. Vantieghem S, Knaepen B (2011) Numerical simulation of maghetohydrodynamic flow in a toroidal duct of square cross-section. Int $\mathrm{J}$ Heat Fluid FI 32: 1120-1128.

12. Krasnov D, Zikanov O, Rossi M, Boeck T (2010) Optimal linear growth in magnetohydrodynamic duct flow. J Fluid Mech 653: 273-299.

13. Hoque M, Alam M (2013) Effects of Dean number and curvature on fluid flow through a curved pipe with magnetic field. Procedia Eng 56: 245-253.

14. Sarris IE, Zikos GK, Grecos AP, Vlachos NS (2006) On the limits of validity of the low magnetic Reynolds number approximation in MHD naturalconvection heat transfer. Num Heat Tr B-Fund 50: 157-180

15. Tanahashi T, Oki T (1995) Numerical analysis of natural convection of thermo-electrically conducting fluids in a square cavity under a uniform magnetic field. JSME Int J 38: 374-381.

16. Hatzikonstantinou PM, Bakalis PA (2014) A computational approach for the solution of the MHD and thermal flow of a liquid metal between two horizontal concentric cylinder. Prog Comput Fluid Dy 14: 259-267.

17. Bakalis PA, Hatzikonstantinou PM (2015) MHD formulations for the liquid metal flow in a curved pipe of circular cross section. Comput Fluids 119: $1-12$.

18. Bakalis PA, Hatzikonstantinou PM (2015) Effect of curvature and magnetic field on MHD flow of a liquid metal in a curved annular duct. Int $\mathrm{J}$ Numer Meth FI 25: 1818-1833.

19. Morley NB, Smolentsev S, Munipalli R, Ni MJ, Gao D, Abdou M (2004) Progress on the modeling of liquid metal, free Surface, MHD flows for fusion liquid walls. Fusion Eng Des 72: 3-34 